Rev. Adm. Saúde (On-line), São Paulo, v. 19, n. 77: e183, out. - dez. 2019, Epub 05 nov. 2019 http://dx.doi.org/10.23973/ras.77.183

ARTIGO ORIGINAL

\title{
Percepção da equipe de enfermagem quanto ao controle de infecção em um hospital de ensino
}

Perception of the nursing team regarding infection control in a teaching hospital

Josni Tauffer ${ }^{1}$, Bruna Tais Zack², Manoela Cristina Berticelli ${ }^{2}$, Maria Julia Navarro Kássim², Sabrina Kássia Menegusso Carmello², Débora Cristina Ignásio Alves ${ }^{3}$

1. Enfermeiro. Residente em vigilância em saúde e controle de infecções no Hospital Universitário (HUOP) do Universidade Estadual do Oeste do Paraná (UNIOESTE), Cascavel PR.

2. Enfermeira. Residente em vigilância em saúde e controle de infecções no Hospital Universitário (HUOP) do Universidade Estadual do Oeste do Paraná (UNIOESTE), Cascavel PR.

3. Enfermeira, doutora em enfermagem. Professora adjunta de enfermagem da UNIOESTE, Cascavel PR.

\section{RESUMO}

Introdução: A prevenção e controle de infecções através de medidas que envolvam a qualificação da assistência hospitalar é primordial quando focado no conhecimento e na importância dos assuntos relacionados a essa temática. Frente a isto, o conhecimento e a concepção da equipe de enfermagem sobre o trabalho do controle de infecção auxiliam na aprimoração da qualidade e obtenção dos resultados esperados. Objetivo: Analisar a percepção dos profissionais da equipe de enfermagem quanto ao serviço de controle de infecção em um hospital escola. Métodos: A coleta de dados seguiu a proposta de estudo quantitativo e qualitativo, formadas por questões abertas e fechadas que foram transcritas de forma analítica e elencadas conforme eixos temáticos as quais sofreram análise de Bardin. Os resultados das questões 
fechadas foram descritos por meio do programa Excel com apresentações dos resultados em frequência e porcentagem. Resultados: 17 (85\%) dos profissionais entrevistados eram do sexo feminino; 18 (90\%) responderam que a falta de adesão à higienização das mãos é responsável por maior parte das infecções relacionadas à assistência à saúde na instituição; 14 (70\%) dos profissionais evidenciaram que a grande circulação de profissionais propicia o surgimento de infecções relacionadas à assistência à saúde; 12 (60\%) dos profissionais afirmaram ter conhecimento sobre os índices de infecções relacionadas à assistência à saúde em seu setor; 17 (85\%) afirmam a disponibilização de ferramentas para o controle de infecções relacionadas à assistência à saúde; 16 (80\%) afirmam ter participado em algum momento de treinamentos relacionados ao controle de infecção; 18 (90\%) higienização das mãos e 11 (55\%) observação nos critérios de isolamento, os demais variam entre 8 a $9 \%$ considerando respectivamente importante a esterilização de materiais e utilização de equipamento de proteção individual; 18 (90\%) afirmam conhecer o trabalho do enfermeiro em controle de infecção. Conclusão: $A$ atuação do profissional enfermeiro faz-se necessária, na vigilância da incidência das infecções relacionadas à assistência à saúde e na prestação de cuidados. O conjunto multifatorial e multiprofissional, que engloba desde incentivos financeiros quanto a prevenção continuada e eficaz ao paciente, com a implementação de linguagem horizontal definida por aplicação de práticas e protocolos assistenciais sem desvios de informações contribui significativamente na redução das infecções relacionadas à assistência à saúde.

Palavras-chave: Infecções relacionadas à assistência à saúde; Controle de Infecção; Vigilância em Saúde; Enfermagem.

\section{ABSTRACT}

The prevention and control of infections through measures involving the qualification of hospital care is paramount when focused on the knowledge and importance of issues related to this thematic. Faced with this, the knowledge and conception of the nursing team about the work of infection control helps to improve the quality and obtain the expected results. Objective: To analyze the perception of nursing team regarding hospital infection control service in a school hospital. Methods: The data collection followed the proposal of a quantitative and qualitative study, formed by open and closed questions which was transcribed analytically and listed according to thematic axes which were analyzed by Bardin. The results of the closed questions were described through the Excel program with presentations of results in frequency and percentage.

Results: 17 (85\%) of the professionals interviewed were female. 18 (90\%) answered that lack of adherence to hand hygiene is responsible for most of the healthcare-associated infections in the institution; 14 (70\%) of the professionals showed the large circulation of professionals facilitates the emergence of healthcare-associated infections; 12 (60\%) of the professionals stated that they had knowledge about the healthcare-associated infections indices in their sector; 17 (85\%) affirm the availability of tools for the control of healthcareassociated infections; 16 (80\%) reported having participated in some training 
related to infection control; 18 (90\%) hands hygiene and 11 (55\%) Note in the isolation criteria, the others vary from 8 to $9 \%$ considering, respectively, material sterilization and use of individual protection equipment; 18 (90\%) stated that they knew the nurse's job in infection control. Conclusion: Nursing professional performance is necessary, in the surveillance of the incidence of healthcareassociated infections and in the provision of care. The multifactorial and multiprofessional set, from financial incentives to continuous and effective prevention to the patient, with the implementation of horizontal language defined by the application of practices and protocols assists without deviations of information contributes significantly to the reduction of healthcare-associated infections.

Keywords: Health-care-related infections; Infection Control; Health Surveillance; Nursing.

\section{INTRODUÇÃO}

As comissões de controle de infecção hospitalar $(\mathrm{CClH})$ foram instituídas pela Lei Federal no 9431 de 1997 em caráter obrigatório a todos os hospitais em território nacional, as quais são pautadas pelas diretrizes de normas e práticas à prevenção e o controle de infecções hospitalares pela Portaria № 2616 de 12 de maio de 1998. A composição do serviço é formada por membros executores que representam a $\mathrm{CClH}$, dos quais são encarregados da execução das ações programadas de controle das infecções no âmbito hospitalar. Esses membros executores devem ser no mínimo, 02 técnicos de nível superior da área de saúde para cada 200 leitos ou fração deste número com carga horária diária, mínima, de 06 horas para o enfermeiro e 04 horas para os demais profissionais. Um dos membros executores deve ser, preferencialmente, um enfermeiro ${ }^{1,2}$.

No tocante as evidências quanto aos custos com tratamento e problemas diretos e indiretos aos pacientes em relação as infecções relacionadas à assistência à saúde (IRAS), sempre foram evidenciadas como um dos grandes problemas de saúde pública no Brasil. Diante ao exposto, medidas pautadas na segurança do paciente vem cada vez mais tomando força nas discussões governamentais, quanto ao ressaltar a importância da atuação da CCIH como um órgão assessor com autoridade máxima atuando diretamente nas questões práticas e institucionais junto as direções gerais e administrativas, que visam proporcionar melhorias à assistência prestada ${ }^{3,4}$.

A atuação direta dos profissionais de saúde no que se diz respeito as medidas de prevenção as IRAS configuram-se por medidas multiprofissionais e interdisciplinares. Dado a isto, a importância da ênfase na formação dos 
profissionais quanto a magnitude em que configuram-se as estratégias pertinentes ao trabalho da realizado pela $\mathrm{CClH}$, transfere a capacidade de trabalhar nas diretrizes que compõem a qualidade do serviço com conhecimento e habilidades praticas, tal ênfase, estruturam a sua competência na assistência à saúde, promovendo qualidade contribuindo para transformações de muitas realidades ${ }^{4,5}$.

Os processos educativos acerca da temática contribuem para atuação dos profissionais em pontos chaves trabalhando acerca da prevenção das IRAS. Isto acontece quando os processos educativos disseminam o conhecimento através dos profissionais da saúde, todavia a baixa adesão por parte das equipes muitas das vezes não atinge os objetivos almejados, e por fim a necessidade de educação permanente e auditoria afim de monitorar as ações na prática faz-se necessário por parte das equipes do Serviço de Controle de Infecção Hospitalar (SCIH) ${ }^{6,7}$.

A equipe de enfermagem tem papel imprescindível no controle das IRAS, garantindo qualidade na assistência prestada contribuindo para garantia de saúde ao indivíduo. A partir desta temática, objetivou-se através deste estudo analisar o conhecimento dos profissionais da equipe de enfermagem acerca da atuação do SCIH na instituição de saúde em questão.

\section{MÉTODOS}

Estudo descritivo, quantitativo e qualitativo desenvolvido em um hospital de ensino localizado no Oeste do Paraná com referência para alta complexidade para trauma de aproximadamente 220 leitos nos setores de enfermaria ${ }^{8}$.

Os critérios estabelecidos abrangeram pelo menos 01 enfermeiro da unidade e 01 técnico ou auxiliar de enfermagem. O número total de participantes foi de 10 enfermeiros e 10 profissionais técnicos e auxiliares de enfermagem.

O questionário foi aplicado entre junho e julho de 2019, o qual foi composto por questões abertas e fechadas de múltipla escolha, que compunham dados referentes ao perfil do profissional entrevistado e conhecimentos sobre a temática do estudo. A fim de preservar as informações pessoais dos profissionais que participaram da pesquisa, foi previamente apresentado o estudo com seus principais objetivos e assinatura do Termo de Consentimento Livre Esclarecido (TCLE) com dados pertinentes aos pesquisadores com instruções sobre o uso das informações.

O questionário foi dividido em 02 blocos de perguntas, o bloco 01 abordava 0 conhecimento do profissional entrevistado acerca da temática, já o bloco 02 abordava a percepção profissional acerca da educação e o suporte físico e material para medidas de controle das IRAS.

O preenchimento do questionário foi feito no mesmo dia da entrega conforme a disponibilidade de cada entrevistado, sem nenhum tipo de influência por parte do entrevistador. 
Posteriormente, os dados quantitativos foram analisados de forma estatística e tabulados em programa Microsoft Excel com apresentações dos resultados em forma percentual e absoluta. Os dados das questões abertas qualitativas foram redigidos em programa Microsoft Word conforme cada eixo proposto afim de organizar a discussão de formas sistemática. Os discursos foram categorizados de acordo com o método proposto por Bardin, que consiste nas seguintes fases a) organização da análise; b) codificação; c) categorização; d) tratamento dos resultados, inferência e a interpretação dos resultados. A organização da análise explora o material, trata os resultados e interpreta-os. A codificação transforma os dados brutos do texto em uma representação do conteúdo e a categorização direciona o conteúdo dos dados analisados ${ }^{9}$.

O presente trabalho seguiu os aspectos éticos atendendo as questões do Comitê de Ética e Pesquisa (CEP) sob o CAEE no 50066815.8.0000.0107 e sob o Parecer ํㅜ 1.447.806, projeto pelo qual conta com a abrangência de estudos voltados para "boas práticas em saúde: segurança do paciente e qualidade da assistência de enfermagem prestada".

\section{RESULTADOS}

Conforme Tabela 1 podemos observar os resultados sobre o perfil dos profissionais de enfermagem entrevistados conforme as variáveis: sexo; formação;

Tabela 1. Caracterização da equipe de enfermagem entrevistada no período de inquérito.

\begin{tabular}{lcc}
\hline Perfil da equipe de enfermagem & N & $\%$ \\
\hline Sexo & & \\
\hline Feminino & 17 & 85 \\
Masculino & 3 & 15 \\
\hline Formação profissional & & \\
\hline Aux. enfermagem & 2 & 10 \\
Téc. enfermagem & 7 & 35 \\
Enfermeiro & 11 & 55 \\
\hline Regime de trabalho & & \\
\hline Estatutário & 12 & 60 \\
Terceirizado & 7 & 35
\end{tabular}


Tempo de atuação na instituição

\begin{tabular}{lrr}
\hline$>10$ anos & 9 & 45 \\
1 a 10 anos & 10 & 50 \\
$<1$ ano & 1 & 5 \\
\hline TOTAL & 20 & $\mathbf{1 0 0}$
\end{tabular}

Com base no perfil dos entrevistados, o seguinte estudo evidenciou que 17 $(85 \%)$ dos profissionais entrevistados eram do sexo feminino, ao qual confrontado com estudo que mostra o perfil dos profissionais de enfermagem do Brasil, sem destoar com os dados apresentados que configuram atualmente sendo em média $85 \%$ dos profissionais do sexo feminino ${ }^{10}$.

Com relação ao setor em que o profissional é lotado, 6 (30\%) dos funcionários atuam na Unidade de Terapia Intensiva Adulto (UTI), 3 (15\%) no Setor de Pronto Socorro (PS), 2 (10\%) dos funcionários estão lotados igualmente no Setor de Ortopedia; Setor de Educação Permanente e Central de Material Esterilizado (CME).

\section{DISCUSSÃO}

No bloco 1 de questões, a pergunta de número 01, que abordava 0 conhecimento sobre IRAS e medidas de controle foi "Pra você quais são as condições que propiciam as IRAS na instituição em geral?", os resultados foram: $18(90 \%)$ responderam que a falta de adesão à higienização das mãos é responsável por maior parte das IRAS na instituição; 14 (70\%) dos profissionais evidenciaram que a grande circulação de profissionais propiciam o surgimento de IRAS; 13 (65\%) dos profissionais afirmam que a falta de conscientização dos profissionais sobre controle de IRAS é um dos fatores contribuintes para o seu surgimento.

Em um estudo sobre a introdução da temática IRAS no curso de graduação em enfermagem, aponta um déficit na introdução do conhecimento sobre controle de infeção e IRAS, o estudo observou embora a temática esteja introdução em um plano pedagógico durante o curso ainda não é abordado de maneira contínua nos quatro anos do curso de enfermagem ${ }^{4}$. O ensino e aprendizagem deveria ser voltado para bases sobre infecções, porque toda e qualquer realização de procedimentos seja ele diagnósticos ou terapêuticos necessitam dos profissionais desenvolverem medidas de prevenção e controle de infecções.

Tal conhecimento proporciona a aplicabilidade no cotidiano profissional, pois auxiliar na vigilância oportuna, possibilitando as medidas de prevenção. Isso foi evidenciado através de um estudo desenvolvido em Gana - África O̧cidental, 
$103(53,8 \%)$ pacientes tinham algum conhecimento sobre IRAS, sendo que 52 $(28,4 \%)$ informados por um profissional de saúde, em comparação com 63 $(88,7 \%)$ dos profissionais de saúde que estavam bem informados sobre IRAS ${ }^{11}$.

Podemos considerar as falas de alguns profissionais que mais aproximaram-se da resposta:

"Toda infecção adquirida em decorrência da prestação de cuidados em saúde, indiferente do ambiente (hospital, ambulatório, domicílio etc.)." (Profissional 01)

"São infecções decorrentes do processo de trabalho, em decurso de diversos fatores, dentre eles, o que tem mais evidência é a falta de adesão à higienização das mãos." (Profissional 16)

"É toda infecção causada pela assistência ao paciente onde teve descuido na manipulação das precauções padrões, cuidados com cateteres e higienização das mãos." (Profissional 20)

Algumas das respostas não atenderam o contexto da temática, dais quais podemos destacar:

"Quando o paciente entra na unidade hospitalar com algum tipo de vírus ou bactéria e adquire outros tipos de bactérias dentro da unidade hospitalar." (Profissional 13)

"Um conjunto de microrganismos patogênicos em um mesmo ambiente, ocasionando riscos a vida." (Profissional 19)

"É uma doença que se dissemina entre os pacientes devido à falta de higienização adequada das mãos, uso adequado de EPI" (Profissional 17)

Neste bloco, foram identificadas na questão de número 02 as falas dos profissionais sobre as principais dificuldades encontradas em seu setor ao fim de controle de infecção, sendo que a maioria das falas pontuaram questões como: quantitativo de funcionários para uma assistência adequada; falta de insumos e dificuldades referentes a fazer-se cumprir protocolos assistenciais, destas podemos destacar algumas das falas:

"Falta de adesão dos profissionais aos protocolos (práticas/medidas assistenciais que contribuem para prevenção das IRAS”. (Profissional 1)

"Torneiras inadequadas, fluxo grande de pessoas principalmente de acadêmicos que muitas vezes não recebem orientações nenhuma das rotinas etc." (Profissional 02)

“Sobrecarga de trabalho, falta de funcionários." (Profissional 19)

"O meu setor é responsável pelo treinamento dos funcionários de todo o hospital, no entanto, há uma falta de adesão dos protocolos 
institucionais e bastante resistência para participação dos treinamentos ofertados." (Profissional 17)

"Grande circulação de estagiários, e residentes não atentos as precauções necessárias, porém não se generaliza nem todos tem as mesmas atitudes, mas alguns mesmo quando são notificados fazem pouco caso. Falta de insumos adequados." (Profissional 15)

Um relatório publicado em 2011 pela Organização Mundial da Saúde (OMS) pontua o ônus causado pelas IRAS, abordando questões pertinentes a promoção e prevenção em saúde das quais ressaltam as condições ideais para efetiva vigilância dessas infecções sendo a falta de recursos humanos, a disponibilização de produtos para saúde adequados e em quantitativo ideal além da educação e responsabilização dos profissionais envolvidos na assistência, os principais motivos para o controle destas infecções ${ }^{12}$.

Quando questionados sobre o seu conhecimento a respeito dos índices de IRAS no seu setor na questão de número 03, 12 (60\%) dos profissionais afirmaram que possuíam conhecimento sobre eles. Estudos apontam que a qualificação profissional surte efeito quando o trabalho é voltado para epidemiologia e vigilância das IRAS, dado que o registro dos dados pontuando a incidência do agravo pode estabelecer ações direcionadas ao seu controle. Em contrapartida à estudos que pontuam esta vigilância com característica retrospectiva não eficaz nas ações de controle da infecção por considerar que o problema já está instalado e a percepção da melhoria ser de maneira gradativa $^{13}$.

A respeito do bloco 2, ao qual aborda educação continuada e logística acerca do controle de infecção, quando questionados sobre as condições adequadas disponíveis em seu setor para o controle de infecção, pela questão de número 05, 17 (85\%) dos profissionais pontuaram positivamente.

Recursos para implementações de novas tecnologias, disponibilização de materiais, uma formação profissional adequada principalmente aos profissionais envolvidos na prevenção das IRAS, a educação e o treinamento representam um componente importante para a implementação precisa das recomendações, Fatores comportamentais também foram identificados como contribuintes para o sucesso das intervenções, estabelecendo pontos chave para melhoria da qualidade prestada, por fim a prevenção das infecções ${ }^{14}$.

Sob justificativa, alguns dos profissionais citaram alguns itens disponíveis em seu setor, tendo consonância a grande maioria.

"Disponibilidade de materiais estéreis para procedimentos invasivos; dispensadores de álcool/sabão para lavagem das mãos; produtos para desinfecção de superfícies”. (Profissional 1)

“Pias, álcool, antissépticos, vestimentas." (Profissional 02)

"Orientação sobre o controle de infecção, álcool gel disponível." (Profissional 09) 
"Pia para lavagem das mãos, álcool em gel, disponibilidade de desinfetantes, box separados." (Profissional 14)

Na questão de número 06 "Existem ações coletivas de prevenção em sua instituição de trabalho afim de controle de infecção?”, 18 (90\%) pontuaram que seu setor possui ações a fim de controle de infecção.

Com a mesma temática, a questão 07 "Nos últimos 02 anos você recebeu treinamento referentes ao controle de infecção?”, 16 (80\%) dos funcionários responderam que participaram em algum momento de treinamentos relacionados ao controle de infecção, se resposta positiva, os participantes descreveram alguns dos treinamentos já tomados, os quais podemos verificar nos excertos a seguir:

"Higienização das mãos. Limpeza/desinfecção de superfície; processos de esterilização de materiais." (Profissional 01)

“Prevenção de PAV, higienização oral." (Profissional 06)

"Treinamento sobre higienização oral, manipulação de cateter e ventilador." (Profissional 15)

Vários estudos pontuam a educação sobre higienização das mãos uma das mais relevantes no que se diz respeito a prevenção. A educação dos profissionais relacionadas à utilização de dispositivo somente quando necessário usando técnicas assépticas e a remoção precoce dele contribuem também para prevenção das IRAS. A implementação de um grupo de intervenções baseadas em evidências conhecidas como bundle, apresenta reduções drásticas nas taxas de infecções em comparação com uma intervenção implementada isoladamente ${ }^{15}$.

Na questão de número 08, que era de múltipla escolha, "Pra você qual é a ação na assistência prestada que mais auxilia no controle de infecção?", 18 $(90 \%)$ consideraram que a higienização das mãos era importante e 11 (55\%) a observação nos critérios de isolamento, as demais alternativas variam entre 8 a $9 \%$ que consideraram respectivamente importante a esterilização de materiais e utilização de equipamentos de proteção individual (EPI). A importância relatada pelos profissionais entrevistados é evidenciada por estudos que comprovam esses dados, os quais demonstram que a higiene das mãos é uma prática importante na redução da transmissão de agentes infecciosos. Outras medidas incluem o uso adequado de antibióticos e diretrizes de apoio laboratorial junto com melhorias científicas no diagnóstico de problemas de saúde relacionados a doenças infecciosas ${ }^{16}$.

A questão de 09 "Você conhece o trabalho do enfermeiro dentro do serviço de Controle De Infecção Hospitalar (SCIH)? Se sim, discorra sobre sua importância?" aponta o conhecimento dos entrevistados sobre a atuação do profissional enfermeiro no setor de $\mathrm{SCIH}$, sendo 18 (90\%) dos entrevistados que responderam positivamente a questão. Podemos analisar as respostas que mais aproximaram-se da temática na transcrição das falas: 
"Vigilância; educação; para os processos de infecção e constituir ações de extrema importância e relevância para prevenção e controle das IRAS na instituição." (Profissional 01)

"Sua importância está associada a qualidade e a segurança da assistência prestada do paciente, uma vez que sem um controle e vigilância dos casos de infecção não há como realizar o controle e políticas para melhoria do cuidado do paciente e do profissional de saúde." (Profissional 05)

"Entendo que cabo ao enfermeiro da CCIH a vigilância, acompanhamento e educação (em parceria com o setor de educação permanente) de todo o hospital no sentido de prevenir casos de IRAS, acompanhar e orientar os profissionais nos casos já vigentes. Além de estar sempre vigilante, alerta para todas as possíveis situações." (Profissional 08)

Entretanto, alguns participantes mostraram-se desinformados quanto a atuação do profissional enfermeiro no $\mathrm{SClH}$.

“Controle de paciente colonizado." (Profissional 04)

"Fiscalizar materiais, vencidos." (Profissional 13)

"Enfermeiro é importante pois possui habilidades de trabalho em equipe organização de trabalho e capacidade de agrupar ações". (Profissional 02)

Uma revisão de literatura aponta diferentes estudos sobre a introdução dos enfermeiros na prevenção e controle de infecções. Enfermeiros dedicados ao programa de controle de infecção atuam como um elo entre sua própria área clínica e a equipe de enfermagem assistencial, aumentando a conscientização sobre prevenção e controle de infecção. Foram identificados quatro temas centrais para o papel dos enfermeiros no controle de infeção sendo eles: atuação como modelo e defensor visível, formador de indivíduos e na prevenção e controle de infecções aplicado a prática, comunicador local e apoio na auditoria e vigilância em saúde. No mesmo estudo são elencadas várias atividades desenvolvidas pelo enfermeiro bem como; realizar vigilância de infecções, monitorar práticas de prevenção e controle, auxiliar na detecção precoce de surtos, melhorar a prática clínica no nível da enfermaria, agir como um modelo e ajudar na pesquisa e auxílio epidemiológico das IRAS na instituição. ${ }^{17}$

\section{CONCLUSÃO}

O SCIH ativo e atuante em vigilância das IRAS é de longe a maior alternativa para redução das infecções em todo o mundo. A percepção e a sensibilização de todos os profissionais envolvidos na prestação de cuidados diretos e indiretos ao paciente contribui significativamente na qualidade, prevenção e promoção em saúde. 
Como peça chave no controle de infecção, a atuação do profissional enfermeiro faz-se necessária, tanto através da vigilância na incidência das IRAS quanto na prestação de cuidados. A educação continuada dos profissionais é uma das ferramentas que mais auxilia em questões como a prevenção das IRAS, pois enfatizam na conscientização dos profissionais que prestam assistência direta ao paciente.

Um dos grandes fatores aliados na prevenção de IRAS é o conjunto multifatorial que engloba desde incentivos financeiros quanto a prevenção continuada e eficaz por todos os profissionais de saúde, sendo esta estabelecida pela implementação de linguagem horizontal definida por aplicação de práticas e protocolos assistências sem desvios de informações.

Exemplo qual podemos ressaltar é a introdução de tecnologias que minimizem os riscos ao paciente, como procedimento menos invasivos possíveis e produtos eficazes que contribuam para a recuperação do paciente e que proporcionem facilidade para equipe de saúde em sua aplicabilidade.

A necessidade de mais estudos que correlatem a percepção dos profissionais de saúde e principalmente de enfermagem, evidenciando os problemas encontrados frente ao controle de infecção e pontuando melhorias aos serviços, faz-se necessária.

\section{REFERÊNCIAS}

1. BRASIL. Ministério da Saúde. Agência Nacional de Vigilância Sanitária. Diretrizes e normas para a prevenção e o controle das infecções hospitalares: Portaria no 2616/98. Brasília (DF), 1998. [citado 23 jul. 2012] Disponível em: http://www.anvisa.gov.br/legis/portarias/2616 98.htm.

2. BRASIL. Ministério da Saúde (BR). Agência Nacional de Vigilância Sanitária. Dispõe sobre a obrigatoriedade da manutenção de programa de controle de infecções hospitalares pelos hospitais do país: lei oㅡ 9431/1997. Brasília (DF), 1997. [citado 23 jul. 2012]. Disponível em: http://www.anvisa.gov.br/legis/leis/9431 97.htm.

3. Dutra GG et al. Nosocomial infection control: role of the nurse. Revista de Pesquisa: Cuidado é Fundamental Online, [s.l.], v. 7, n. 1, p.2159-2168, 1 jan. 2015. Universidade Federal do Estado do Rio de Janeiro UNIRIO. http://dx.doi.org/10.9789/2175-5361.2015.v7i1.2159-2168.

4. Giroti SKO, Garanhani ML. Infections related to health care in nurses' education. Revista da Rede de Enfermagem do Nordeste, [s.I.], v. 16, n. 1, p.64-71, 3 abr. 2016. Rev Rene - Revista da Rede de Enfermagem de Nordeste. http://dx.doi.org/10.15253/2175-6783.2015000100009.

5. Monteiro TS. Infecção Hospitalar: Visão Dos Profissionais da Equipe de Enfermagem. Revista de Epidemiologia e Controle de Infecção, [s.l.], v. 5, n. 
2, p.1-5, 31 ago. 2015. APESC - Associação Pro-Ensino em Santa Cruz do Sul. http://dx.doi.org/10.17058/reci.v5i2.5665.

6. Massaroli AM, Jussara G, Massaroli R. Educação Permanente para o aperfeiçoamento do Controle de Infecção Hospitalar: revisão integrativa. Saúde \& Transformação Social Health \& Social Change, Florianópolis, v. 5, n. 1, p.7-15, jul. 2015. Disponível em:

http://incubadora.periodicos.ufsc.br/index.php/saudeetransformacao/article/v iew/2500/3642. ISSN 2178-7085. Acesso em: 15 abr. 2019

7. Silva AC, Rodrigues LMS, Souza MMT, Bibiano RS. A enfermagem frente à educação permanente na prevenção e no controle da infecção hospitalar. Revista PróUniverSUS. 2014 Jul./Dez.; 05 (2): 05-10.

8. Cadastro Nacional de Estabelecimentos de Saúde CNES. [citado em 2016 set. 08]. Disponível em:

http://cnes2.datasus.gov.br/cabecalho reduzido.asp?VCod Unidade $=41048$ $\underline{02738368 .}$.

9. Bardin L. Análise de conteúdo. São Paulo: Loyola; 2010.

10. Machado $\mathrm{MH}$ et al. Características gerais da enfermagem: O Perfil sócio demográfico. Revista Oficial do Conselho Federal de Enfermagem, Rio de Janeiro, v. 6, n. 1, p.9-14, nov. 2015. Disponível em:

<http://revista.cofen.gov.br/index.php/enfermagem/article/view/686/296>> Acesso em: 25 abr. 2019.

11. Ocran I, Tagoe DNA. Knowledge and attitude of healthcare workers and patients on healthcare associated infections in a regional hospital in Ghana. Asian Pacific Journal of Tropical Disease, [s.l.], v. 4, n. 2, p.135-139, abr. 2014. Elsevier BV. http://dx.doi.org/10.1016/s2222-1808(14)60330-3.

12. WORLD HEALT ORGANIZATION. GENEVA. (Org.). Report on the burden of endemic health care-associated infection worldwide. 2011. Disponível em: $<$ https://apps.who.int/iris/bitstream/handle/10665/80135/9789241501507 en g.pdf;jsessionid=A7F2D473FA41C90B6DFC99AC7B48F049? sequence $=1>$. Acesso em: 25 abr. 2019.

13. Oliveira HM, SILVA, Rodrigues CP, Lacera RA. Policies for control and prevention of infections related to healthcare assistance in Brazil: a conceptual analysis. Revista da Escola de Enfermagem da Usp, [s.I.], v. 50, n. 3, p.505-511, jun. 2016. Fap UNIFESP (SciELO). http://dx.doi.org/10.1590/s0080-623420160000400018.

14. Birgand G. et al. Overcoming the obstacles of implementing infection prevention and control guidelines. Clinical Microbiology And Infection, [s.I.], v. 21, n. 12, p.1067-1071, dez. 2015. Elsevier BV. http://dx.doi.org/10.1016/i.cmi.2015.09.005.

15. Vincent H. Prevention of Health Care-Associated Infections. Am Fam Physician, Orlando, v. 90, n. 6, p.377-382, nov. 2014. Disponível em: 
https://www.aafp.org/afp/2014/0915/p377.pdf. Acesso em: 25 abr. 2019.

PMID: 25251230.

16. Almeida S. Health Care-Associated Infections (HAls). Journal of Emergency Nursing, [s.l.], v. 41, n. 2, p.100-101, mar. 2015. Elsevier BV. http://dx.doi.org/10.1016/i.jen.2015.01.006.

17. Dekker $\mathrm{M}$ et al. Infection control link nurses in acute care hospitals: a scoping review. Antimicrobial Resistance \& Infection Control, [s.l.], v. 8, n. 1, p.2-13, 28 jan. 2019. Springer Nature. http://dx.doi.org/10.1186/s13756-019$\underline{0476-8}$.

Recebido: 03 de setembro de 2019. Aceito: 05 de novembro de 2019

Correspondência: Josni Tauffer. E-mail: josnitauffer@hotmail.com

Conflito de Interesses: os autores declararam não haver conflito de interesses.

(C) This is an Open Access article distributed under the terms of the Creative Commons Attribution License, which permits unrestricted use, distribution, and reproduction in any medium, provided the original work is properly cited 